\title{
Identifikation oder Reflexion? Zur frühen deutschen Rezeption von Diderots Erzählung Les deux amis de Bourbonne
}

Der Hinweis auf die Bedeutung Diderots für den deutschen Sturm und Drang ist ein fester Topos in der einschlägigen Forschung. ${ }^{1}$ Als ein Kronzeuge wird in diesem Zusammenhang immer Goethe angeführt, der seiner frühen Auseinandersetzung mit der französischen Literatur in Dichtung und Wahrheit einen längeren Abschnitt gewidmet hat. Wie er schreibt, sei er zu Beginn der 1770er Jahre für die Fortsetzung seines Jura-Studiums nicht zuletzt deshalb nach Straßburg gegangen, weil er die französische Sprache seit seiner Kindheit geliebt habe (vgl. DuW, $522) .^{2}$ Doch schon bald sei es zu einer Ernüchterung gekommen. Offenbar wurde er immer wieder von Franzosen auf die Unzulänglichkeit seines an sich guten, wenn auch ein bisschen eigenwilligen Französisch hingewiesen, so dass ihm die Lust am französischen Gespräch verging. Den deutschen Landsleuten, mit denen er befreundet war, ging es nicht anders, und so kapselte sich dieser Kreis von jungen Männern zunehmend ab. Gesprochen wurde nur noch Deutsch, und man steigerte sich gegenseitig in eine Überhöhung der deutschen Kultur als eine Kultur ungekünstelter Aufrichtigkeit hinein, die hell abstach gegen die - angebliche - gezierte Unnatürlichkeit der Franzosen. Mit altersweiser Selbstironie bemerkt Goethe zu dieser jugendlichen Germanophilie:

Schon früher und wiederholt auf die Natur gewiesen, wollten wir [...] nichts gelten lassen als Wahrheit und Aufrichtigkeit des Gefühls, und den raschen derben Ausdruck desselben.

Freundschaft, Liebe, Brüderschaft,

Trägt die sich nicht von selber vor?

1 Vgl. z.B. Anne Saada: Diderot und der Sturm und Drang, in: Bodo Plachta, Winfried Woesler (Hg.): Sturm und Drang. Geistiger Aufbruch 1770-1790 im Spiegel der Literatur, Tübingen 1997, S. 23-39; oder Roland Mortier: Diderot in Deutschland 17501850 [1954], übers. von Hans G. Schürmann, Stuttgart 1972, v.a. S. 156-168.

2 Hier und im Folgenden wird mit der Sigle DuW und Seitenzahl verwiesen auf Johann Wolfgang Goethe: Aus meinem Leben. Dichtung und Wahrheit, hg. von Klaus-Detlef Müller (= Sämtliche Werke, Briefe, Tagebücher und Gespräche [Frankfurter Ausgabe = FA], Bd. 14), Frankfurt a.M. 1986, S. 522 . 
war Losung und Feldgeschrei, woran sich die Glieder unserer kleinen akademischen Horde zu erkennen und zu erquicken pflegten. Diese Maxime lag zum Grunde allen unsern geselligen Gelagen, bei welchen uns denn freilich manchen Abend Vetter Michel in seiner wohlbekannten Deutschheit zu besuchen nicht verfehlte. (DuW, 526f.)

Für die Jünglinge dieser »kleinen akademischen Horde« hatte die aufgeklärte französische Literatur der bienséance wenig Anziehendes. Sie schien ihnen in Konventionen der sogenannt besseren Gesellschaft gefangen und im Wesentlichen nur noch eine späte Nachlese zur klassischen Literatur der Epoche Ludwig XIV. zu sein. Sie war, wie Goethe schreibt, »bejahrt und vornehm, und durch beides kann die nach Lebensgenuß und Freiheit umschauende Jugend nicht ergetzt werden« (DuW, 527).

Eine Ausnahme gab es freilich für die deutschen Jünglinge unter den zeitgenössischen französischen Schriftstellern, eben Diderot - freilich nicht den Mitherausgeber der Encyclopédie, bei deren Lektüre es dem jungen Goethe zu Mute war, "als wenn man zwischen den unzähligen bewegten Spulen und Weberstühlen einer großen Fabrik hingeht« (DuW, 531), sondern den Theaterautor und den Erzähler. Dieser Diderot konnte für die Stürmer und Dränger gleichsam zum Wahlverwandten - und sogar zum Landsmann - werden: »Diderot war nahe genug mit uns verwandt; wie er denn in alle dem, weshalb ihn die Franzosen tadeln, ein wahrer Deutscher ist. [...] Seine Naturkinder [...], die er mit großer rednerischer Kunst herauszuheben und zu adeln wußte, behagten uns gar sehr, seine wackeren Wilddiebe und Schleichhändler entzückten uns [...]« (DuW, 531f.).

Mit den »Naturkinder[n]« ist, unter anderem, auf die Bedeutung von Diderot als dem Autor des Fils naturel und des Entretien sur le fils naturel für das deutsche Theater verwiesen; eine Bedeutung, die vor allem mit Lessings Übersetzung Das Theater des Herrn Diderot (1760) eingesetzt hatte. ${ }^{3}$ Die Bemerkung zu den »Wilddieben und Schleichhändlern«

3 Vgl. [Denis Diderot:] Das Theater des Herrn Diderot. Aus dem Französischen [von Gotthold Ephraim Lessing], Berlin 1760. Diese Sammel-Übersetzung enthält im ersten Band das Stück Der natürliche Sohn (Le Fils naturel) und die »wahre Geschichte des Stücks« (das heißt den Entretien sur les Fils naturel), im zweiten das Schauspiel Der Hausvater (Le père de famille) und die Abhandlung Von der dramatischen Dichtkunst (De la poésie dramatique). Vgl. auch die kommentierte Neuausgabe: Das Theater des Herrn Diderot. Zweisprachige, synoptische Edition der Diderot-Übersetzung von 1760, hg. von Nikolas Immer und Olaf Müller, St. Ingbert 2014. Vgl. im vorliegenden Band auch den Beitrag von Beate Hochholdinger-Reiterer. 
aber zielt auf Diderots Erzählung Les deux amis de Bourbonne und deren Bedeutung für die deutsche Literatur -, auch wenn hier Goethe eine kleine Ungenauigkeit unterlaufen ist, denn diese Erzählung ist erst 1772, also kurz nach seiner Straßburger Zeit im Druck erschienen. ${ }^{4}$ Das ändert freilich nichts an ihrer Bedeutung für die Sturm und Drang-Generation.

Auf den ersten Blick scheint es auf der Hand zu liegen, was die Faszination von Diderots Erzählung für jene Generation ausmachte: Ihre Helden - Olivier und Felix - stammen aus einfachsten Verhältnissen und sind einander von frühester Kindheit an in unverbrüchlicher Freundschaft verbunden. Sie retten sich gegenseitig mehrfach das Leben, ohne je ein Wort darüber zu verlieren, und demonstrieren immer wieder unter prekärsten Bedingungen ihre Aufrichtigkeit und Ehrlichkeit, wobei diese natürliche Tugendhaftigkeit scharf kontrastiert mit der Verkommenheit der gerichtlichen und kirchlichen Macht-Instanzen, mit denen Olivier und Felix in Konflikt geraten. Bedeutet schon allein Diderots Wahl seiner Protagonisten einen politisch aufgeladenen Verstoß gegen die Ständeklausel - sie sind »des héros absolument neufs « ${ }^{5}$-, gewinnt die Erzählung durch die Kontrastierung der idealen Freundschaft von »Schleichhändlern« mit dem Verhalten der Mächtigen noch zusätzliche politisch-gesellschaftliche Sprengkraft. Sie konnte mithin als Ausdruck einer Rebellion gegen die bestehenden Verhältnisse im Namen der Natur gelesen werden und fand entsprechend bei den sjugendbewegten<, autoritäts- und gesellschaftskritischen Stürmern und Drängern eine begeisterte Aufnahme - während sie in Frankreich partiell verboten wurde. ${ }^{6}$ In ihr kam, wie Goethe rückblickend bemerkte, ein vorrevolutionärer Impuls zum Ausdruck, der ganz allgemein von jener Sozialkritik ausging, die mit den Namen Diderot und Rousseau verbunden wurde: "So war er [Diderot] es denn auch, der, wie Rousseau, von dem geselligen Leben einen Ekelbegriff verbreitete« und damit »eine stille Einleitung zu jenen ungeheueren Weltveränderungen, in welchen alles bestehende unterzugehen schien« mitinitiierte (DuW, 532).

Vgl. dazu Mortier: Diderot in Deutschland (wie Anm. 1), S. 155.

5 Als solche bezeichnet sie Jacques Proust in seiner Einleitung zu Denis Diderot: Quatre Contes, Genève 1964, S. LXXVII.

6 Vgl. zu diesem Verbot Wiebke Röben de Alencar Xavier: Salomon Gessner im Umkreis der Encyclopédie. Deutsch-französischer Kulturtransfer und europäische Aufklärung, Genève 2006, S. 327-332. 
Einige Jahrzehnte nach Goethe hat auch Karl Rosenkranz - als Verfasser der 1866 erschienenen ersten deutschen Diderot-Monographie - von der sozialkritischen Dimension in Les deux amis de Bourbonne gesprochen und, mit direktem Bezug auf Goethe, auf die begeisterte Rezeption dieser Erzählung in Deutschland hingewiesen. Sie gehört für ihn zu einer ganzen Reihe von »sociale[n] Novellen", in denen Diderot »Schicksale aus der Nachtseite der menschlichen Gesellschaft« behandelt habe. ${ }^{7}$ Dabei rückt er auch noch ein stilistisches Element in den Vordergrund, das bereits für den Sturm und Drang wichtig war, für das aber Rosenkranz, als Zeitgenosse des Realismus und Verfasser der $\ddot{A} s$ thetik des Hässlichen (1853), besonders sensibilisiert war: die realistische, schlichte, ungekünstelte Schreibweise Diderots:

Die Wirkung dieser Erzählung [Les deux amis de Bourbonne] in ihrer realistischen, knappen Manier fiel nicht nach Frankreich, sondern nach Deutschland. Goethe berichtet in seinem »Leben«, daß sie auf den strasburger[!] Kreis einen gewaltigen Eindruck machte. Man fühlt bei ihrem Waldleben, ihren Kohlenbrennern, ihren Galgenscenen, ihren Kämpfen mit der Gensdarmerie[!], ihren Leidenschaften, ihrer Wahrheit eines mit Worten kargen Naturausdrucks, schon eine ganz ähnliche Atmosphäre, als sie uns in Schiller's »Räubern« begegnet. $^{8}$

Mit Schillers Räubern (1781) hat Rosenkranz ein Werk genannt, das seither immer wieder erwähnt wird, wenn es um die Frage geht, wo in der deutschen Literatur Spuren von Les deux amis de Bourbonne auszumachen sind ${ }^{9}-$ auch wenn sich Diderots und Schillers Protagonisten in verschiedenen Punkten klar unterscheiden: So stammt Karl von Moor zum Beispiel, ganz anders als Diderots Freunde, aus adligem Hause, und während der Schiller'sche Räuberhauptmann antike Klassiker liest ${ }^{10}$ geht den Freunden Felix und Olivier jede Bildung ab. Rosenkranz’ Bemerkung zur Nähe der Räuber zu Diderots Erzählung könnte

7 Karl Rosenkranz: Diderot's Leben und Werke, Leipzig 1866, Bd. 2, S. 306 (Hervorheb. im Original).

8 Ebd., S. 304.

9 Vgl. z.B. Edmond Eggli: Diderot et Schiller, in: Revue de littérature comparée 1 (1921), S. 68-127, hier S. 86 oder Saada: Diderot (wie Anm. 1), S. 36f. Daneben wird auch immer der Einfluss von Diderot auf den Erzähler Schiller - etwa im Verbrecher aus Infamie - erwähnt. Ebd., S. 37. Kritisch dazu Kathrin Ackermann: Die Rezeption von Diderots Erzählungen in Deutschland und Frankreich, in: Günter Berger, Franziska Sick (Hg.): Französisch-deutscher Kulturtransfer im Ancien Régime, Tübingen 2002, S. 113-143, hier S. 131-133.

10 Bei seinem ersten Auftritt sagt Karl von Moor: »Mir ekelt vor diesem Tintenklecksenden Sekulum, wenn ich in meinem Plutarch lese von großen Menschen.« Friedrich 
also durchaus mit Fragezeichen versehen werden, doch sofern damit einfach auf die unübersehbare Konjunktur von Räuber- und Außenseitergeschichten verwiesen sein soll, die in der deutschen Literatur seit den 1780er Jahren ausgemacht werden kann, ist sie sicher nicht falsch. Und auch Rosenkranz' Hinweis auf Diderots Realismus, der in Deutschland ein vielfältiges Echo finden sollte, ist richtig. Sowohl das inhaltliche als auch das stilistische Element trugen - je mit ihren politischen Implikationen - zur Bedeutung von Les deux amis de Bourbonne für eine sozialkritisch engagierte Literatur in Deutschland bei. Ausgeblendet aber blieb bei Rosenkranz - und fast durchgehend in der deutschen Rezeption seit dem Sturm und Drang - die Frage der Form von Diderots Erzählung. Das heißt, vor lauter Begeisterung über den Inhalt und den dazu passenden knapp-realistischen Stil verlor man die auffallende und komplexe Form der Erzählung aus den Augen; eine Form, die - wie gezeigt werden soll - ihrerseits politische Implikationen hat, und eine Form, die den auf eine Identifikation mit den >Helden angelegten Lektüren zumindest partiell den Boden entzieht. Oder noch einmal anders formuliert: Nimmt man die Form von Diderots Erzählung ernst, wird deutlich, dass die Geschichte von Olivier und Felix so elaboriert perspektiviert wird, dass sich der Fokus - und das heißt auch der politische Fokus - letztlich auf die Meta-Ebene dieser Perspektivierung verschiebt. Es ginge mithin nur sekundär um die Schicksale des unterprivilegierten Freundespaares, primär aber um die Art und Weise, wie diese Schicksale von mehreren, unterschiedlich stark ins Geschehen involvierten Erzählinstanzen geschildert werden. Und indem Diderot in seinem Text vorführt, welche Wirkungen solche verschieden perspektivierte Darstellungen auf Personen innerhalb seines Erzählkosmos haben können, regt er auch das Lesepublikum zur Reflexion über die Wirkungen an, denen es bei seiner Lektüre ausgesetzt ist. Kurz, Les deux amis de Bourbonne ist nicht auf Identifikation, sondern auf Reflexion hin angelegt - womit sich zeigt, dass die deutsche Rezeption dieser Erzählung im Grunde über weite Strecken auf einem Missverständnis beruhte.

Beim Versuch nun, die politische Semantik der Form von Diderots Erzählung stark zu machen, soll im Folgenden zunächst (I.) kurz deren Publikationsgeschichte skizziert werden; zum einen, weil die Textge-

Schiller: Die Räuber. Ein Schauspiel, in: Ders.: Werke und Briefe, hg. von Klaus-Harro Hilzinger u.a., Frankfurt a.M. 1988, Bd. 2, S. 11-160, hier S. 30. 
nese auch Argumente für die Bedeutung der formalen Ebene liefert, zum andern, weil diese Geschichte ein eigenes, irritierend-faszinierendes Kapitel der Diderot-Rezeption in Deutschland ausmacht. Danach soll (II.) die Struktur der Erzählung genauer beschrieben und analysiert werden, um zu zeigen, was in der Regel ausgeblendet blieb in der frühen deutschen Rezeption. Und in einem letzten Abschnitt wird (III.) jene Rezeption noch einmal genauer in den Blick genommen, wobei vor allem eine 1773/74 erschienene Rezension von Johann Jakob Engel besprochen werden soll; eine Rezension, die deshalb von besonderem Interesse ist, weil hier - entgegen der Haupttendenz der Rezeption - allgemeine Ansätze zu einer Berücksichtigung der Bedeutung der Form auszumachen sind; Ansätze, die schon voraus zu deuten scheinen auf die erst im ausgehenden 20. Jahrhundert richtig erfolgte 'Entdeckung< von Diderots politischer Formsemantik. ${ }^{11}$ In der konkreten Auseinandersetzung mit Les deux amis de Bourbonne hat freilich auch Engel nichts gemacht aus dieser Sensibilität für die Form. Damit bestätigt auch diese Ausnahme die Regel, wonach die frühe deutsche Rezeption - ganz im Banne des Erzählstoffes stehend - das politische Potential der Form von Diderots Erzählung übersah.

I.

Am Anfang der Textgenese von Les deux amis de Bourbonne stand nicht die Absicht, eine sozialkritische Erzählung zu schreiben, sondern die Lust an einem literaturkritischen Spiel der Mystifikation: Diderot verbrachte im Sommer 1770 einige Tage in Bourbonne, wo er Madame de Maux und deren Tochter, Madame de Prunevaux, besuchte. ${ }^{12}$ Letztere unterhielt eine Korrespondenz mit Jacques-André Naigeon, der mit Diderot bekannt war und später zum Herausgeber seiner Werke werden sollte. Und im Rahmen dieser Korrespondenz machte sie sich einen Spaß daraus, Naigeon mit erfundenen Geschichten zum Narren

11 Vgl. dazu das Diderot-Kapitel in der wichtigen Studie von Dena Goodman: Criticism in Action. Enlightenment Experiments in Political Writing, Ithaca/London 1989, S. 167-224. Auf Les deux amis de Bourbonne geht Goodmann freilich kaum ein. Vgl. auch die Einleitung zum vorliegenden Band.

12 Vgl. zur Textgenese den Kommentar in Denis Diderot: Euvres complètes, hg. von Herbert Dieckmann u.a. (= DPV), Bd. XII, Paris 1989, S. 415-421. 
zu halten. Diderot aber ließ sich nur allzu gerne dazu überreden, unter der Maske seiner Freundin auch einen Beitrag zu diesem Täuschungsspiel zu leisten - nicht zuletzt auch um zu testen, ob Naigeons Behauptung, er könne schon an wenigen Zeilen erkennen, ob ein Text von ihm stamme, stichhaltig sei. Zugleich nahm Diderot diese Gelegenheit auch wahr, um Kritik an einer gerade erschienenen, exotistisch-idealisierenden Erzählung Jean-François de Saint-Lamberts über eine ideale Freundschaft zwischen zwei Irokesen zu üben. ${ }^{13}$ So entstand die erste Version von Les deux amis de Bourbonne, ${ }^{14}$ deren Protagonisten nicht in einem verklärten fernen Land, sondern mitten unter einfachen Leuten in Frankreich leben, und sich nicht - wie bei Saint-Lambert in gedrechselt-süßlichen Phrasen über ihr Gefühlsleben austauschen, sondern eine Sprache pflegen, die der Kargheit ihrer Lebensumstände entspricht.

Das Täuschungsmanöver gelang gleich doppelt: Weder erkannte Naigeon Diderots Autorschaft, noch hatte er Zweifel an der Faktizität der Geschichte von Olivier und Felix. Und Diderot selber fand so viel Vergnügen an seinem literaturkritischen Mystifikationsspiel, dass er die Erzählung von den beiden Freunden weiter ausarbeitete und sie im Dezember 1770 in Grimms Correspondance littéraire publizierte. Freilich wurde sie damit nur einem sehr exklusiven Leserkreis bekannt, denn die Correspondance zirkulierte nur in handschriftlichen Kopien. Im Druck erschien Les deux amis de Bourbonne erst 1772 - und zwar auf Deutsch. Und ist schon allein dieser Umstand bedeutsam im Kontext der Frage nach der deutschen Diderot-Rezeption, so wird das Phänomen der Erstausgabe noch viel auffälliger, wenn man sieht, wo und durch wen die Erzählung veröffentlicht wurde: Glaubt man Salomon Gessner, so erfuhr Diderot über gemeinsame Bekannte in Paris, dass der in Frankreich überaus populäre Schweizer eine neue Sammlung von Idyllen herausbringen wollte, und bat ihn darum, selber zwei

13 Vgl. Jean-François de Saint-Lambert: Les deux amis. Conte iroquois, Paris 1770. Saint-Lamberts conte ist nur ein Beispiel für eine ganze Welle von damals erschienenen literarischen Werken zur Freundschaftsthematik. Vgl. zu diesem Kontext Michel Delons Kommentar in Denis Diderot: Contes et Romans, hg. von Michel Delon, Paris 2004, S. 1052-1055.

14 Es gibt eine erst 1985 zum ersten Mal publizierte >Urversion< der Erzählung, von der freilich unklar ist, in welchem Verhältnis sie zu den tatsächlich an Naigeon geschriebenen Briefen steht. Sie ist abgedruckt in Diderot: DPV (wie Anm. 12), Bd. 12, S. 422-436. 
Erzählungen zu dem geplanten Band beisteuern zu dürfen. ${ }^{15}$ Diese Chance wollte sich Gessner nicht entgehen lassen, und so kam es, dass ihm Diderot die leicht überarbeitete Geschichte von den zwei Freunden sowie die dialogische Erzählung Entretien d'un père avec ses enfants, ou du danger de se mettre au dessus des lois schickte. Gessner machte sich umgehend - mit der Hilfe von Freunden - an die Übersetzung, und so erschien Die beyden Freunde von Bourbonne tatsächlich zum ersten Mal zusammen mit den neuen Idyllen Gessners. ${ }^{16}$ Im Jahr darauf erschien diese Gemeinschaftspublikation von Gessner und Diderot dann - ebenfalls in Zürich - auch in einer sehr aufwendig gestalteten und von Gessner mit Kupferstichen illustrierten Luxus-Ausgabe auf Französisch. ${ }^{17}$

Über diese beiden Zürcher Ausgaben wurde die Erzählung Diderots einem breiten Publikum bekannt und fand eben das erwähnte begeisterte Echo unter den Vertretern des Sturm und Drang. Es ergibt sich mithin der reizvoll-irritierende Befund, dass die Diderot'schen »Schleichhändler« gleichsam im Windschatten der Gessner'schen Hirten in den literarischen Diskurs eingetreten sind; dass die proto-realistische Geschichte Diderots aus den Niederungen der Gesellschaft des Ancien Régime zusammen mit den antikisierend-gekünstelten Idyllen Gessners in ein und demselben Buch vereint waren; ein Befund, der seit dem Erscheinen des Gemeinschaftswerks immer wieder für kritische und irritierte Kommentare gesorgt hat. ${ }^{18}$

Nun war für die Leser der Gessner'schen Ausgabe von Les deux amis de Bourbone freilich nicht mehr erkennbar, dass diese ursprünglich sozusagen aus einem literaturkritischen Gesellschaftsspiel hervorgegangen

15 Vgl. dazu Gessners Ausführungen »An den Leser!« in: [Salomon Gessner:] Moralische Erzaehlungen und Idyllen von Diderot und S. Gessner, Zürich 1772, S. 133f. Vgl. zur Geschichte der Publikation Röben de Alencar Xavier: Salomon Gessner (wie Anm. 6), S. 259-300.

16 Denis Diderot: Die beyden Freunde von Bourbonne. Eine Erzaehlung, in: Gessner/ Diderot: Moralische Erzaehlungen und Idyllen (wie Anm. 15), S. 135-170. Vgl. zu Gessners Übersetzung auch den Beitrag von Boris Previšić im vorliegenden Band.

17 [Salomon Gessner, Denis Diderot:] Contes moraux et nouvelles idylles de D... et Salomon Gessner, Zuric 1773; in dieser französischen Ausgabe setzte Gessner die Erzählungen Diderots, anders als in der deutschen Ausgabe, an den Anfang. Es ist nicht ohne Ironie, dass die Geschichte der armen Schlucker Felix und Olivier diese Luxusausgabe eröffnete, die nur für ein privilegiertes Publikum erschwinglich war und die mit einer ausführlichen Subskribentenliste versehen war, in der typographisch klar differenziert wurde zwischen Angehörigen regierender Adelsfamilien und anderen Bestellern. Vgl. ebd., ohne Paginierung.

18 Vgl. zusammenfassend den (den Gegensatz zu Recht auch relativierenden) Kommentar von Michel Delon in Diderot: Contes et Romans (wie Anm. 13), S. 1050 . 
war. Hatte Grimm in einer Vorbemerkung zur Version in der Correspondance littéraire noch - in mehr oder weniger anonymisierter Form - auf jenen Anlass verwiesen, ${ }^{19}$ so gab es in der Zürcher Erstausgabe keine entsprechenden paratextuellen Hinweise mehr, und innerhalb der Erzählung waren die letzten Spuren des Namens von Madame de Prunevaux getilgt: War sie bei Grimm noch »Madame de $\mathrm{P}^{* * *}{ }_{«}$, war sie jetzt nur noch $»$ Madame de ${ }^{* * *}{ }_{\ll}$. Dadurch wurde die Erzählung aus ihren biographischen Zusammenhängen herausgelöst - weshalb man auch bei ihrer Lektüre konsequent von »Madame de ${ }^{* * *}$ « und nicht von »Madame de Prunevaux« sprechen sollte. ${ }^{20}$ Und mit dieser Herauslösung aus dem Mystifikationsspiel wurde wahrscheinlich auch der auf Identifikation hin angelegten Lektüre in Deutschland Vorschub geleistet. Denn es konnte vergessen gehen, dass es in diesem Text im Grunde nur in zweiter Linie um das tatsächliche Schicksal von Olivier und Felix geht, in erster aber um die Reflexion über den erzählerischen Umgang mit diesem Schicksal. Um nun aber zu zeigen, inwiefern auch die von Gessner veröffentlichte Version auf diese Reflexion hin angelegt ist, muss der Aufbau der Erzählung genauer beschrieben und analysiert werden.

II.

Zu Beginn von Les deux amis de Bourbonne ist nicht klar, wer spricht. Erkennbar ist nur, dass sich die erzählende Person offenbar dort befindet, wo die Protagonisten der Geschichte einst gelebt haben: »Es lebten hier ein Paar Leute, man könnte sie den Orest und Pylades von Bourbonne nennen; der eine hieß Olivier, der andre Felix« $(\mathrm{D}, 135) .^{21}$ Das deiktische "hier « lässt vermuten, dass sich die Erzählinstanz an ein Gegenüber wendet, das schon weiß, wo sie sich befindet; etwa so, wie in einer Korrespondenz zwischen zwei Bekannten. Und tatsächlich erhärtet sich dieser Verdacht, wenn im zweiten Abschnitt unversehens

19 Diese Vorbemerkung ist abgedruckt in Diderot: DPV (wie Anm. 12), S. 437.

20 Das ist zum Beispiel auch in der ansonsten differenzierten Interpretation von Baron der Fall. Vgl. Konstanze Baron: Diderots Erzählungen. Die Charaktergeschichte als Medium der Aufklärung, Paderborn 2014, S. 309-332.

21 Hier und im Folgenden wird mit der Sigle D und Seitenzahl verwiesen auf Diderot: Die beyden Freunde von Bourbonne (wie Anm. 16). 
ein »Brüderchen« $(\mathrm{D}, 136)$ angesprochen wird, an das der Bericht über das Schicksal der beiden Freunde adressiert ist. In dieser Grundstruktur ist für den Eingeweihten noch die Spur des Mystifikationsspiels erkennbar, das Madame de Prunevaux, unterstützt von Diderot, mit Naigeon gespielt hat. Freilich darf dieses biographische Hintergrundwissen bei der Lektüre der Erzählung eben nicht vorausgesetzt werden. Vielmehr muss die inszenierte Verunsicherung darüber, wer eigentlich erzählt, ernst genommen werden.

Nach dem zitierten Eingangssatz beginnt die Erzählinstanz mit der Geschichte der beiden Freunde, die »am gleichen Tage, in dem gleichen Hause und von zwoo Schwestern gebohren« (D, 135) wurden und wie Geschwister aufwuchsen, da die eine Mutter bei der Geburt starb. Sie liebten einander "so wie man sein Daseyn, sein Leben genießt, ohne daran zu denken« (D, 135). Und dieses Moment der Nicht-Reflexivität prägt dann auch ihr weiteres Leben. Ohne zu zögern setzen sie ihr Leben füreinander ein, sowohl als Knaben wie dann auch als Jünglinge bei der Armee. Und als sie sich beide - »von ohngefehr« (D, 137) - in dasselbe Mädchen verlieben, kommt es zu keiner Eifersucht: »Der erste, der die Leidenschaft seines Freundes gewahr ward, entfernte sich: Es war Felix. Olivier heyrathete; und Felix, des Lebens überdrüssig, ohne zu wissen warum, stürzte sich in allerley gefährliche Gewerbe, und ward zuletzt ein Schleichhändler« (D, 138). Bald wird er verhaftet und zum Tode verurteilt, dann aber im letzten Moment von Olivier im wörtlichen Sinne >herausgehauen< - wobei es auch in der Syntax sozusagen Schlag auf Schlag geht:

Vor Wuth ausser sich steht Olivier auf, versetzt mit seinem Stocke dem Richter Coleau [der ihm erst mit fataler Verzögerung gesagt hat, dass Felix gerade hingerichtet werden soll] einen ungeheuern Streich auf das Genicke, der ihn fast todt auf den Boden streckt; läuft gegen dem[!] Richtplatz; kömmt, schlägt auf den Henker; schlägt auf die Gerichtsdiener; bringt den Pöbel auf, der ohnedies schon über solche Hinrichtungen erbittert war. Es fliegen Steine. Felix wird befreyt und entkömmt. (D, 139f.)

Olivier verliert den Freund aus den Augen und flieht auf eigene Faust, ist allerdings so schwer verletzt, dass er bei der Rückkehr zu seiner Familie verstirbt - ohne viel Aufhebens darum zu machen, wie es seinem Charakter entspricht: »Kaum hatte er noch Zeit, seinem Weibe zu sagen: Weib, komm her, dass ich dich herze: Ich sterbe! Aber der Narbigte [Felix] ist gerettet!« $(\mathrm{D}, 140)$. 
An dieser Stelle bricht das erzählende Ich ab und geht nun ohne rechte Überleitung darauf ein, wie es vom Schicksal der beiden Freunde erfahren habe. Es sei nämlich eines Abends in Begleitung spazieren gegangen und habe »vor einer Strohhütte« (D, 140) eine Frau mit vier kleinen Kindern getroffen. Wie sich herausstellt, handelte es sich dabei um Oliviers Witwe, und diese habe die Geschichte ihres verstorbenen Manns erzählt.

Ex post wird mithin klar, dass das, was man zuvor gelesen hat, sozusagen eine bearbeitete Fassung der Erzählung von Oliviers Witwe ist, und mit dieser verschriftlichten Variante einer ursprünglich mündlichen Erzählung will das schreibende Ich seinem Adressaten - dem »Brüderchen« oder »Freund « (D, 141) - demonstrieren, dass, wie es abschließend bemerkt, »Hoheit der Seele, und erhabne Eigenschaften, allen Ständen und allen Ländern zukommen; daß oft einer in Dunkelheit stirbt, dem es nur an einem andern Schauplatze fehlte; und daß man in einer Strohhütte oder bey den Irokesen zween Freunde finden kann.« (D, 141f.)

Nach dieser Quintessenz, in der die Spitze gegen Saint-Lamberts Conte iroquois unübersehbar ist - und die in späteren Ausgaben noch um den Hinweis auf ein Beispiel unerschütterlicher Treue unter sizilianischen Räubern ergänzt wurde ${ }^{22}$-, folgt im Text eine mit drei Sternchen markierte Zäsur, bevor das erzählende Ich in einem zweiten Brief der offenbar vom "Brüderchen« in seiner Antwort auf den ersten Brief aufgeworfenen Frage nach dem weiteren Schicksal von Felix nachgeht. Und jetzt erfährt man auch, wer die Begleitung des Ichs ist. Es ist »Mama« (D, 142), die, wie das Ich berichtet, vorgeschlagen habe, nicht, wie ursprünglich geplant, beim örtlichen Pfarrer Papin Erkundigungen über Felix' Schicksal einzuholen, sondern »bey dem Subdelegat Aubert«

22 Vgl. für diese ergänzte Passage Diderot: DPV (wie Anm. 12), Bd. 12, S. 443. Diderot hat dieses Beispiel übernommen aus [Johann Hermann von Riedesel:] Voyage en Sicile et dans la Grande Grèce, Lausanne 1773, S. 167. Die deutsche Originalausgabe war zwei Jahre zuvor in Zürich erschienen: [Johann Hermann Riedesel:] Reise durch Sizilien und Großgriechenland, Zürich 1771; das Beispiel findet sich dort auf S. 170f. Diderot fügte die entsprechende Passage wohl kurz nach Erscheinen der französischen Ausgabe von Riedesels Reisebericht in seine Erzählung ein; im Druck erschien die ergänzte Version von Les deux amis de Bourbonne aber erst postum; zum ersten Mal 1795 in einer Werkausgabe Gessners, dann auch 1798 in der Diderot-Werksausgabe von Naigeon. Heutige Ausgaben der Erzählung drucken in der Regel die ergänzte Version ab. 
(D, 142). ${ }^{23}$ Der habe auch tatsächlich einen Bericht verfasst, und dieser wird dann im Brief des erzählenden Ichs über mehrere Seiten hinweg wörtlich in Anführungszeichen zitiert - wobei zum ersten Mal richtig klar wird, dass jenes Ich eine "Madame« ist, denn als solche wird es von Aubert angesprochen. Damit ist endlich deutlich, dass es sich bei dem "wir«, von dem zuvor die Rede war, um Mutter und Tochter handelt. Wie schon aus den ersten Abschnitten von Auberts Bericht hervorgeht, ist auch dieser keine Erzählung aus erster Hand. Vielmehr beruft sich der Subdelegat auf die Auskünfte einer Köhlerin, bei deren Familie Felix nach seiner Flucht vom Schafott untergekommen war. Kaum war Felix wieder bei Kräften, so hat Aubert erfahren, überredete er den Köhler, mit ihm auf die Suche nach Olivier zu gehen, denn noch wusste Felix nichts von dessen Tod. Auf dem Weg wurden die beiden aber in einen Kampf mit den Landjägern der »Marechaussee« (D, 143) verwickelt, bei dem der Köhler starb, und als Felix dessen Leiche zur Familie zurückbrachte kam es zu einer herzzerreißenden Szene; einer tableau-artigen Szene, die Gessner übrigens zu einer Illustration inspirierte, deren Expressivität markant absticht von der klassischen Harmonie der Kupferstiche, die er für seine Idyllen geschaffen hat (Abb. 1):

»Bey der Thüre stand er [Felix] still, legte den Leichnam vor seine [eigenen] Füsse, und setzte sich, den Rücken an einen Baum gelehnt, und das Gesicht gegen den Eingang der Hütte gekehrt, nieder. Dieß war der Anblick, der auf die Kohlenbrennerin wartete, wenn sie ihre Hütte öffnen würde. [...] sie [die Kohlenbrennerin] sieht, schreyt, fällt rückwärts zu Boden. Ihre Kinder laufen hinzu, sehen, schreyen, wälzen sich auf ihren Vater, wälzen sich auf ihre Mutter. Durch den Lerm und durch das Geschrey ihrer Kinder wieder zu sich selbst gebracht, zerrauft sich die Kohlenbrennerin die Haare, zerfleischt ihre Wangen. Felix, am Fusse seines Baumes unbeweglich, die Augen geschlossen, den Kopf rückwärts hängend, schreyt mit erstorbner Stimme: Tödtet mich!« (D, 147f.)

${ }^{23}$ Zum Amt des Subdelegaten heißt es im Kommentar zu Diderot: DPV (wie Anm. 12), Bd. 12, S. 444, Anm. 13: »Les subdélégués aidaient les intendants dans leurs fonctions de surveillance et de renseignement. «Die Intendanten waren in der königlichen Verwaltung des Ancien Régime für bestimmte Provinzen zuständig. 


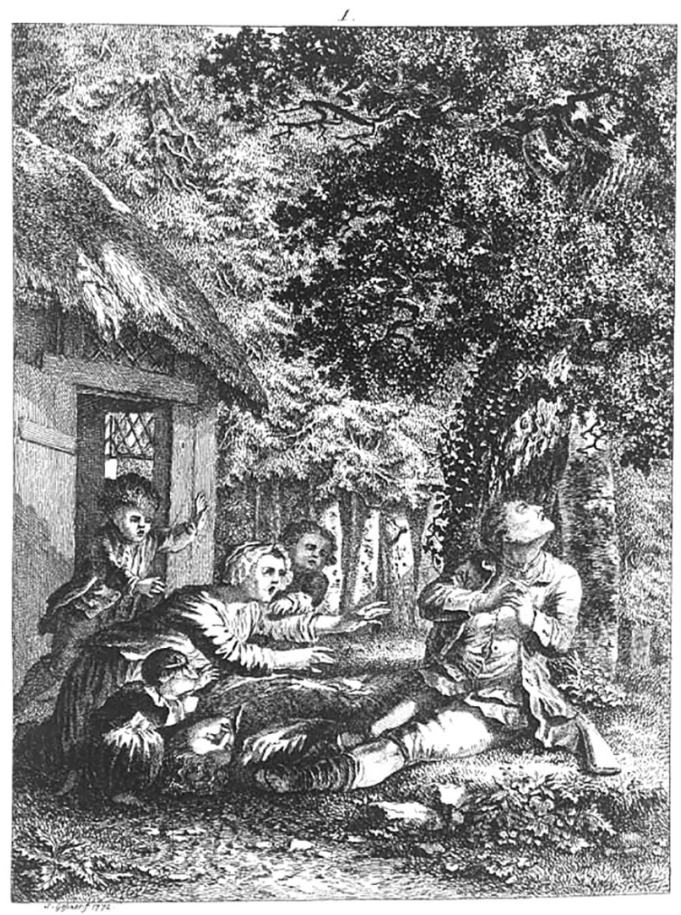

Abbildung 1: Salomon Gessners Illustration zu Denis Diderot: Les deux amis de Bourbonne, in: Contes moraux et nouvelles idylles de D... et Salomon Gessner, Zuric 1773, ohne Paginierung (Bayerische Staatsbibliothek digital).

Nach drei Tagen der Trauer machte sich Felix dann, zusammen mit der Köhlerin und ihren Kindern, wieder auf die Suche nach Olivier. So kamen sie nach Bourbonne, wo sie von dessen Witwe erfuhren, dass er tot war - worauf Felix, von Trauer und Schuldgefühlen geplagt, für längere Zeit in einen wahnsinnsähnlichen Zustand verfiel. Der Aufenthalt in Bourbonne war für ihn freilich gefährlich, denn noch immer wurde nach ihm gefahndet, und so tauchte er wieder unter, nachdem er noch schnell die Hochzeit eines Sohnes von Olivier mit einer Tochter des Köhlers arrangiert hatte - was, nebenher bemerkt, Fragen nach der Chronologie aufwirft. ${ }^{24}$ Später erfuhr ein vornehmer Herr von seinem

${ }^{24}$ Im ersten Brief von Madame ist von den kleinen Kindern der Witwe die Rede. Da überrascht es, dass nun eines dieser Kinder bereits heiraten kann. Vgl. allgemein 
Schicksal, hatte Mitleid und sorgte dafür, dass er begnadigt wurde womit übrigens ein positiv gezeichneter Angehöriger des Adels in die Geschichte eingeführt wird. ${ }^{25}$ Felix kam zurück und trat als Wildhüter in den Dienst jenes Herrn, ließ sich dann aber in einem Streit mit einem Nachbarn zu einer Gewalttat provozieren - die nur ein Beispiel unter mehreren ist für die gesellschaftlich durchaus nicht unproblematische Unreflektiertheit von Felix ${ }^{26}$-, so dass er ins Gefängnis kam. Freilich kam er auch diesmal frei, denn des »Stockmeisters Tochter verliebte sich in ihn und bahnte ihm den Weg zur Flucht« (D, 158), die ihn letztlich bis nach Preußen führte, wo er ins Garderegiment des Königs eintrat. Die Familien des Köhlers und Oliviers habe er aber nicht vergessen: Noch immer schicke er ihnen regelmäßig Geld, wie Aubert vermerkt - ein Umstand, der beim Leser freilich die Frage aufwirft, weshalb sich denn Mutter und Tochter überhaupt an Aubert und nicht noch einmal an die Witwe Oliviers gewandt hatten, um Felix' Geschichte zu erfahren. Denn ganz offensichtlich stand diese ja noch in Kontakt mit Felix. Doch diese Frage bleibt unbeantwortet; genau wie auch die, weshalb Aubert seinem Bericht zusätzlich noch ein verschlossenes Schreiben des Pfarrers Papin beilegt, obschon er - wie aus seinem Kommentar hervorgeht - vermutet, dass dieser Brief keineswegs in seinem Sinne abgefasst ist: »Was er enthält, weiß ich nicht; ich fürchte aber sehr, der arme Priester, dessen Verstand ein bisgen schmal, und das Herz ziemlich schlimm ist, schreibe Ihnen von Olivier und Felix nach seinen Vorurtheilen« (D, 159). Und diese Befürchtung ist nur zu berechtigt, wie der an dieser Stelle in vollem Wortlaut in die Erzählung eingerückte Brief zeigt. Offenbar hatte Papin erfahren, dass sich »Madame« (und deren Mutter) für die Geschichte von Olivier und Felix interessierten - wofür er gar kein Verständnis hat: »Ich weiß nicht, Madame, [...] was für Antheil Sie an dem Schicksal der zween Strassenräuber nehmen können, die alle ihre Schritte in dieser Welt mit Blut besudelt haben« (D, 160). Es gebe zwar Leute, »die von diesen

zur »chronologie [...] quelque peu troublée« den Kommentar in Diderot: DPV (wie Anm. 12), Bd. 12, S. 419.

25 Auch die adlige »Madame« zeichnet sich zunächst durch ihr Mitleid aus. Später erweist sie sich allerdings nicht als zuverlässige Helferin.

${ }^{26} \mathrm{Zu}$ Recht weist Baron darauf hin, dass die Gewalttätigkeit von Felix und Olivier konstitutiv ist für deren Charakter; sie gehört insofern, gleichsam als Schattenseite, mit zur Treue und Tugendhaftigkeit der beiden Freunde. Vgl. Baron: Diderots Erzählungen (wie Anm. 20), S. 331. 
zween Menschen, als von Mustern einer ausserordentlichen Freundschaft« redeten (D, 160f.), doch wer, wie Felix und Olivier »der Kirche und ihren Dienern« keine "Ehrfurcht« entgegenbringe und sich dem »Gesetz des weltlichen Oberherrn« nicht unterwerfe, verdiene kein Mitleid. Wohl solle man den »Armen« helfen, doch allein die Kirche wisse, wer der Unterstützung würdig sei, und deshalb solle »Madame« eine allfällige Spende an ihn überweisen.

Nach diesem Brief - der als Anhang auch noch zum zweiten Schreiben von »Madame« gehört ${ }^{27}$ - kommt es in der Erzählung zu einem weiteren Bruch. Denn nun übernimmt eine übergeordnete Erzählinstanz die Regie und berichtet von der Wirkung von Papins Brief auf »Madame«, die nun »Frau von ${ }^{* * *} *(\mathrm{D}, 162)$ genannt wird: Sie habe Aubert für seinen Bericht gedankt und zugleich Papin »ihre Allmosen « geschickt - mit dem folgenden Brief:

Mein Herr! ich bin Ihnen für Ihren weisen Rath sehr verbunden. Ich gestehe Ihnen, die Geschichte dieser beyden Leute hatte mich gerührt; und Sie werden mir beystimmen, daß das Beyspiel einer so ausserordentlichen Freundschaft recht dazu gemacht war, ein gutes und empfindliches Herz zu verführen: Aber Sie haben mich zurechtgewiesen; und ich hab' es begriffen, daß es besser ist, christlichen und unglücklichen Tugenden Beystand zu leisten, als bloß natürlichen und heidnischen. Ich bitte Sie, die geringe Summe, die ich Ihnen sende, anzunehmen, und sie nach einer klügern Mildthätigkeit auszutheilen, als die Meinige nicht ist.

Ich habe die Ehre zu seyn, u.s.f. (D, 163)

Wie der Erzähler nach diesem eingerückten - und uns als Lesende einigermaßen irritierenden ${ }^{28}$ - Brief bemerkt, könne man sich leicht

27 Es ist demnach nicht richtig, wenn Baron, ebd., S. 313, behauptet, mit dem Brief Papins beginne »die Ebene des Kommentars«; diese beginnt erst nach Papins Brief.

28 Es überrascht, wie leicht der Pfarrer Frau von *** umstimmen kann, die damit in keinem guten Licht erscheint. Zu diesem Meinungsumschwung wurden verschiedene Thesen formuliert. Recknagel z.B. meint, Diderot zeige anhand von Frau von *** in didaktisch-aufklärerischer Absicht, wie man sich durch einen Geistlichen nicht manipulieren lassen sollte. Anne-Christin Recknagel: Diderots Erzählung Les deux amis de Bourbonne. Eine Analyse unter produktions- und rezeptionsästhetischen Gesichtspunkten, in: Peter Brockmeier (Hg.): Französische Literatur in Einzeldarstellungen, Bd. 1, Stuttgart 1981, S. 401-420. Das legt nahe, dass Frau von ***s Urteil ursprünglich ein unverfälschtes gewesen wäre. Doch das ist, wie erwähnt, nicht der Fall. Vgl. dazu auch Baron: Diderots Erzählungen (wie Anm. 20), S. 315f. Baron ihrerseits meint, Diderot führe in Frau von *** das Beispiel einer unzuverlässigen Freundin vor, das die Moral, wonach wohlhabende Menschen keine wahren Freunde sein können, illustriere. Weil sie etwas zu verlieren habe, arrangiere sie sich mit der Machtinstanz des Pfarrers. Es 
vorstellen, dass Oliviers Witwe und Felix ${ }^{29}$ nichts von der erwähnten »geringen Summe« erhielten. Felix aber sei später gestorben. - Und nach einer erneuten, mit drei Sternchen gekennzeichneten Zäsur im Text holt der Erzähler zur berühmt gewordenen theoretischen Coda aus, in der er zunächst das Wesen der »historischen Erzählung « - in Abgrenzung von der "wunderbaren« und der »scherzhaften« Erzählung - erörtert. Der historische Erzähler habe, wie er ausführt, »die strenge Wahrheit zum Gegenstande« (D, 166). Diese Wahrheit ist bei ihm allerdings nicht - wie oft geschehen - als Wahrheit eines Abbildrealismus zu verstehen, wie aus der unmittelbaren Fortsetzung der Argumentation hervorgeht: »Er [der historische Erzähler] will, daß man ihm glaube; will interessiren, rühren, hinreissen, erschüttern, die Haut schauern, und Thränen fliessen machen: [...]«(D, 166f.). Es geht ihm also letztlich nicht um den Bezug auf die reale Welt, sondern vor allem um die Wirkung auf die Rezipienten seiner Erzählung. Diese Wirkung könne, wie er fortfährt, allein durch »Beredsamkeit und Poesie« hervorgebracht werden; zwei Künste, die freilich durch ihre Tendenz, zu »vergrössern, [zu] erweitern [und zu] übertreiben« das Misstrauen der Leser erregen würden. Deshalb müsse der Erzähler kleine Details einbauen, die dieses Misstrauen zerstreuen indem sie, wie man mit einem Anachronismus sagen könnte, einen »Realitätseffekt ${ }^{30}$ hervorrufen:

Er wird in seine Erzählung kleine Umstände die genau mit der Sache verbunden sind, so einfältige, so natürliche, und dabey doch so schwer zuerfindende[!] Züge streu'n, daß ihr [die Leser] euch genöthigt fühlen werdet, bey euch selbst zu sagen: mein Treu, das ist wahr! Dergleichen Dinge erfindt man nicht. So wird er die Vergrösserung der Beredsamkeit und Poesie wieder gut machen; so wird die Wahrheit der Natur das Blendwerk der Kunst verbergen [...]. (D, 167)

Die Wahrheit der historischen Erzählung ist mithin eine bloß konstruierte Wahrheit, deren Konstruktions-Charakter mit Hilfe von Details vernebelt wird, die >Natürlichkeit< und >Wahrheit< vortäuschen. Der

überzeugt jedoch nicht recht, ihr (zunächst) von Mitleid und Anteilnahme geprägtes Verhältnis zu Felix und Olivier (respektive zu deren Geschichte) im Begriff der Freundschaft zu fassen. Vgl. ebd., v.a. S. 314.

29 An dieser Stelle unterlief Gessner ein Übersetzungsfehler. Er spricht von den »Wittwen Olivier und Felix « (D, 164), obschon Felix ja nie verheiratet war. Im Original heißt es: »On pense bien que la veuve Olivier \& Félix n'eurent aucune part aux aumônes de Madame de ***." Denis Diderot: Les deux amis de Bourbonne, in: Gessner/ Diderot: Contes moraux et nouvelles idylles (wie Anm. 17), S. 19.

30 Vgl. Roland Barthes: L’Effet du réel, in: Communications 11 (1968), S. 84-89. 
entsprechende Erzähler aber erweist sich damit zugleich als »wahrhaft und [als] ein Lügner« (D, 168).

Nach diesen poetologischen Ausführungen - die oft, aber letztlich fälschlicherweise als Antizipation realistischer Programme des 19. Jahrhunderts gelesen worden sind ${ }^{31}$ - folgt dann noch, ohne weiter ausgeführte Überleitung, ein Abschnitt, in dem der Erzähler kurz die Moral seiner Geschichte expliziert; die Moral, wonach »es selten eine vollkommene, wahre Freundschaft geben kann, als zwischen Menschen die nichts haben « $(\mathrm{D}, 170)$ - eben so, wie Felix und Olivier oder die Köhler-Familie.

Diese >Moral der Geschichteく, mit der Die beyden Freunde von Bourbonne endet, irritiert nun aus mehreren Gründen; zunächst durch die Abruptheit und Beiläufigkeit, mit der sie an die poetologischen Bemerkungen angehängt wird: »Und jetzt noch ein bisgen Moral auf ein bisgen Poetick. Das steht so gut! - $(\mathrm{D}, 169)$. Dann irritiert sie aber auch durch ihren recht schlichten und eindeutigen Gehalt, der erstens die problematischen Seiten der unreflektierten, »animalische[n] [...] Freundschaft« $(\mathrm{D}, 137)^{32}$ zwischen Felix und Olivier ausblendet und der zweitens in einem auffallenden Missverhältnis steht zur komplexen Form der vorangegangenen Erzählung. Musste die Geschichte von Felix und Olivier wirklich so ausführlich und vor allem auf diese verwickelte Art und Weise erzählt werden, um die Moral auf den Punkt zu bringen, dass nur arme, durch keinen Besitz korrumpierte Leute einander wahre Freunde sein können? Kaum. Vielmehr muss die komplexe Form der Erzählung als Aufforderung ans Lesepublikum verstanden werden, über die Voraussetzungen der - vermeintlich - abschließenden Moral der Geschichte nachzudenken. Damit wird aber auch deutlich, dass die auf den ersten Blick ganz unverbunden nebeneinander stehenden poetologischen und moralischen Ausführungen in der Coda in Wirklichkeit eng aufeinander bezogen sind. Denn die Voraussetzungen für moralische Urteile und Entscheidun-

31 Vgl. dazu Delons Kommentar in Diderot: Contes et Romans (wie Anm. 13), S. 1050: »Diderot construit son conte selon une logique de l'effet et de la croyance qui n'a que peu à voir avec ce que le réalisme du siècle suivant définira comme une enquête et un exposé, c'est à dire une >littérature du vrai<, selon la formule du Mercure français de $1826[\ldots] . .$.

32 Als solche bezeichnet die »Frau von ${ }^{* * *}$ « die Freundschaft von Felix und Oliver; bei ihr ist das positiv auf die Unreflektiertheit der beiden Freunde bezogen. Aus anderer Warte betrachtet liegt in eben dieser Animalität auch die gesellschaftliche Problematik jener Freundschaft. 
gen sind - wie eben auch durch das Spiel der Erzählperspektiven vorgeführt wird - Erzählungen; und genauer noch: standort- und interessengebundene Erzählungen. ${ }^{33}$

Damit soll nun nicht gesagt sein, dass Diderot in Les deux amis de Bourbonne einfach alle Sichtweisen auf das Schicksal der beiden Freunde Olivier und Felix nivellieren und keine Wertungen erkennen lassen würde. So wird etwa der Pfarrer Papin über die Sichtweise, die in seinem Brief zum Ausdruck kommt, deutlich als Inbegriff eines engstirnigen, kaltherzigen Geistlichen charakterisiert und karikiert. Aber es ist keineswegs so, dass die Perspektiven, die in Frau von ${ }^{* * *}$ s Briefen oder im Bericht Auberts zum Ausdruck kommen, einen unverstellten Blick auf die >Wirklichkeit< oder >Wahrheit bieten würden.

In ihrem ersten Brief an das »Brüderchen« stützt sich Frau von ***, wie gesehen, ganz auf die Erzählung von Oliviers Witwe. Als direkt Betroffene hat diese - aus naheliegenden Gründen - eine eigene Sicht auf die Dinge, und sie erzählte ihre Geschichte zudem aus einer ganz konkreten Motivation heraus, denn schon bevor sie zu erzählen begonnen hatte, hatte ihr Frau von *** eine mögliche Unterstützung in Aussicht gestellt. Es ging für die Witwe also darum, die vornehme Dame für ihr Schicksal einzunehmen und ihr Mitgefühl nach Möglichkeit zu steigern. Das ist ihr offensichtlich auch gelungen, wobei sie in Frau von *** eine Zuhörerin fand, die von ihrer Disposition und ihren Lektüre-Erfahrungen her zu einer Idealisierung und Überhöhung des Gehörten tendierte. So ist zum Beispiel der Vergleich der Freundschaft von Felix und Olivier mit jener von Orest und Pylades - im ersten Satz der Erzählung - zweifellos ein Zusatz der Frau von ***. Durch diese Parallelisierung der Freunde mit dem mythologischen Freundespaar par excellence ${ }^{34}$ wird deutlich, wie das Schicksal von Felix und Olivier

33 Vgl. zu dieser Engführung von Poetik und Moral in Diderots Erzählung auch die wichtigen Ausführungen von Baron: Diderots Erzählungen (wie Anm. 20), S. 305-335.

34 Die Vorbildlichkeit der Freundschaft zwischen Orest und Pylades, die schon ihre Kindheit zusammen verbracht haben, wird zum Beispiel besonders hervorgehoben in Ciceros Gespräch über die Freundschaft: Cicero: Laelius de amicitia. Lateinisch/ deutsch, übers. von Max Faltner, München 1961, S. 33-35 (VII/24). Erwähnt wird dort, wie ein Theaterstück aufgeführt worden sei, in dem Orest und Pylades in einer Schlüsselszene gegenseitig ihr Leben für einander opfern wollten. Das habe zu Szenenapplaus im Theater geführt: »Aufgesprungen waren die Leute sogar, um einer erdichteten Szene Beifall zu spenden: Was hätten sie wohl erst getan, wäre die Begebenheit Wirklichkeit gewesen? (ebd., S. 35) Man könnte sagen, dass Diderot mit der konstruierten Wirklichkeit seines "conte historique« in gewissem Sinne dieser Frage nachgeht: Was macht jemand, der die Geschichte der 'wirklichen< Freundschaft zwi- 
in der Nacherzählung der vornehmen und gebildeten Dame stilisiert wird. Diese möchte in den beiden Freunden ein Idealpaar sehen, das sich ohne jede Eitelkeit und Eigenliebe gegenseitig geholfen hat. Das zeigt sich auch etwa in einer Passage, in der sie auf eine Episode aus der gemeinsamen Zeit der Freunde bei der Armee zu sprechen kommt: »Bey der Armee ward Olivier einst im Gefecht von einem Säbelhieb bedroht, der ihm den Kopf spalten sollte: Maschinenmässig stellte sich Felix vor den Hieb, und behielt davon eine Narbe. Man behauptet, er sey auf diese Wunde stolz gewesen; ich glaube es aber nicht« (D, 137). Man könnte auch sagen: Sie will es nicht glauben, weil dieser Stolz nicht zum Idealbild der von ihr imaginierten >Naturkinder<-Freundschaft passt.

Ähnliches wie vom ersten Brief der Frau von *** ließe sich auch vom Bericht des Subdelegaten Aubert sagen, der seinerseits auf den Aussagen der Köhlerswitwe beruht, die zweifellos nicht weniger standortgebunden erzählte als die Witwe Oliviers, mit der sie in ihrem Schicksal vereint war. So zeigt sich zunächst innerhalb der Erzählung, wie moralisches Urteilen auf der Grundlage von »contes historiques« erfolgt, die nicht darauf angelegt sind, eine objektive Wirklichkeit wiederzugeben, sondern - um noch einmal aus der Coda zu zitieren - zu »interessiren, rühren, hinreissen, erschüttern, die Haut schauern, und Thränen fliessen zu machen « (D, 166). Und indem die Leser von Les deux amis de Bourbonne Zeugen dieser Verquickung von (perspektivischem) Erzählen und Moral werden, sollten sie nicht nur erkennen, wie eng die vermeintlich ganz unverbundenen Ausführungen in der Coda zur »Poetick« einerseits und zur »Moral« andererseits zusammenhängen, sondern eben auch, wie ihr eigenes moralisches Urteil durch das Erzählen konditioniert wird. Sie sind mithin eben aufgefordert, jenseits der bloBen Identifikation mit den Protagonisten, über die sich Frau von ***, der Subdelegat Aubert und der Pfarrer Papin austauschen, über jene Konditionierung zu reflektieren.

schen Olivier und Felix hört oder liest? - Wenn Frau von *** das antike Freundespaar ins Spiel bringt, denkt sie nur an deren vorbildliche Freundschaft. Was sie ausblendet, ist der tragische Hintergrund von Orests Schicksal als Muttermörder. Etwas von dieser Tragik ist, selbstverständlich in abgewandelter Form, auch im Schicksal von Felix erkennbar, der sich schuldig fühlt für den Tod von Olivier und dem Köhler und darüber vorübergehend halb wahnsinnig wird. Zudem ist eine der beiden Mütter von Olivier und Felix - man erfährt nicht welche (vgl. D, 135) - bei der Geburt ihres Kindes gestorben. 
III.

In der frühen deutschen Rezeption von Les deux amis de Bourbonne ging das erwähnte Moment der Reflexion freilich - wie eingangs angedeutet - verloren. Für die Vertreter des Sturm und Drang stand die Begeisterung für die - angeblich - tugendhaften, aufrichtigen und ehrlichen »Wilddiebe und Schleichhändler« (Goethe) ganz im Vordergrund, und die durch keinen Besitz korrumpierte Beziehung zwischen Olivier und Felix konnte jenseits ihrer erzählerischen Perspektivierung zum Inbegriff einer wahren Freundschaft werden. Und auch noch da, wo diese Freundschaft im ausgehenden 18. Jahrhundert als haltlose Schwärmerei kritisiert wurde, wurde vorausgesetzt, dass Diderots Erzählung ein auf Identifikation hin angelegtes Manifest für eine Freundschaft im Zeichen der Natürlichkeit sei. So werden die Protagonisten Diderots etwa in einer Abhandlung aus den 1780er Jahren in einem Atemzug mit Goethes Werther als Beispiele für »Schwärmerey« genannt: „[W]enn Olivier nach Rheims läuft, um seinen Freund Felix, der als ein Schleichhändler gehangen werden soll, noch einmal zu sehen [...], wenn er [...] seinen Freund mit Gefahr und Verlust seines eignen Lebens befreyt; wenn Werther seinen qualvollen Leiden unterliegt, und seinen Leiden mit seinem Leben zugleich ein Ende macht; so ist das [...] Schwärmerey [...].“35

35 Schack [= Jacques] Hermann Ewald: Ueber das menschliche Herz. Ein Beytrag zur Charakteristik der Menschheit, Erfurt 1789, S. 372f. Ewald scheint sich mit seiner Kritik an der Schwärmerei auch von seiner eigenen Vergangenheit als Mitglied des Göttinger Hainbundes distanziert zu haben. Als solches hatte er selber einen Oden-Band veröffentlicht: Schack Hermann Ewald: Oden, Gotha 1772. Diesen rezensierte der junge Goethe in den Frankfurter Gelehrten Anzeigen und meinte, Ewald stehe »zwar die Barden- und Odengrammatik so ziemlich zu Dienste [...], aber weder Bardenimagination noch Odengefühl, noch selbst Odenmusik. Große Worte, freche Metaphern und verschlungne Perioden in ein Sylbenmaß zusammen geschraubt, das macht noch keine Ode." Goethe: FA (wie Anm. 2), Bd. 18, S. 94. (Im Kommentar ebd., S. 1099, wird der rezensierte Oden-Band fälschlicherweise dem dänischen Dichter Johannes Ewald zugeschrieben.) Es scheint fast, als hätte sich Ewald diese vernichtende Kritik zu eigen gemacht, wenn er in der zitierten Passage, unmittelbar vor der Erwähnung der Freundschaftsschwärmerei von Felix und Olivier schreibt: »[...] wenn der Dichter sich in der Begeisterung hinauf schwingt in das graue Zeitalter der alten Barden, und, unbekannt mit ihren Sitten, Gebräuchen und Gesängen, in Bärenfell gehüllt, mit Eichenlaub gekrönt, aus Felsenklüften heult, und unserm Jahrhundert Gesänge vortönt, die weder er, noch sonst ein Mensch versteht; [...] so ist das [...] Schwärmerey.« Ewald: Ueber das menschliche Herz (Anm. 35), S. 372. 
Neben der Frage, ob Felix und Olivier nun vorbildliche Freunde seien oder nicht, ging also die Frage nach der Form von Diderots Erzählung unter; ein Umstand, der nicht zuletzt dadurch anschaulich illustriert werden kann, dass Les deux amis de Bourbonne in Deutschland auch in einer mehrfach aufgelegten Bearbeitung erschien, in der nicht nur das Spiel der Perspektiven zugunsten einer einsinnigen Erzählweise aufgehoben, sondern auch die ganze Coda weggekürzt wurde. ${ }^{36}$

Nun gibt es allerdings eine Ausnahme unter den frühen deutschen Stimmen zu Diderots Erzählung: Johann Jakob Engel, der später bekannt werden sollte durch seine zweibändige Mimik (1785), widmete der Luxus-Ausgabe von Diderots und Gessners Contes moraux E nouvelles Idylles in der Neuen Bibliothek der schönen Wissenschaften und der freyen Künste eine längere Besprechung, in der er insbesondere auf Diderots Erzählungen eingeht. ${ }^{37}$ Einleitend weist er dort auf die unbefriedigende Situation hin, dass diese zwar in Deutschland gelobt, aber nicht wirklich in ihrer Eigentümlichkeit kritisch gewürdigt worden seien, und er thematisiert auch die so unterschiedliche Rezeption in Deutschland und Frankreich:

Unsre deutschen Journale [...] haben die Diderotischen Erzehlungen nur angezeigt und gelobt, nicht beurtheilt. Von den französischen haben einige sie sehr unwürdig behandelt. Sie haben es Gessners übergroßer Gefälligkeit zugeschrieben, daß er so mittelmäßige Sächelchen seines Freundes in seine Werke aufnehmen wollen. Bey uns hingegen wäre man lieber über die Ehre erschrocken, die Diderot durch ihre Mittheilung Geßnern erwiesen. So gewiß ist es, daß ein Prophet nirgends weniger gilt, als in seinem Vaterlande. $(\mathrm{E} 1,99 \mathrm{f} .)^{38}$

${ }^{36}$ Diese Bearbeitung erschien unter dem Titel »Freundschaft« in einer vom bekannten Berliner Pädagogen und Publizisten Gedike herausgegebenen Anthologie französischer Literatur: Friedrich Gedike (Hg.): Französisches Lesebuch für Anfänger [1785], 3. Aufl., Berlin 1789, S. 96-102. Vgl. auch die in gleichem Sinne bearbeitete deutsche Version, die unter dem Titel »Olivier und Felix " erschien in: Heinrich Karl Gutmann (Hg.): Magazin von moralischen Erzählungen für alle Fälle der Sittenlehre, alphabetisch geordnet, 1. Bd., Wien 1808, S. 409-414.

37 Vgl. zu dieser Besprechung auch Mortier: Diderot in Deutschland (wie Anm. 1), S. 161-168; für eine Einordnung in den weiteren Zusammenhang von Engels poetologischen Überlegungen den Kommentar und das Nachwort von Voss in Johann Jakob Engel: Über Handlung, Gespräch und Erzählung, hg. von Ernst Theodor Voss, Stuttgart 1964.

38 Hier und im Folgenden wird mit der Sigle E1 und Seitenzahl verwiesen auf Johann Jakob Engel: [Rezension von] Contes moraux \& nouvelles Idylles de D... \& Salomon Gessner, à Zuric, chez l'auteur, MDCCLIII, in: Neue Bibliothek der schönen Wissenschaften und der freyen Künste 15/1 (1773), S. 99-111. 
Wie Engel vermutet, habe diese unbefriedigende Situation mit dem ungewöhnlichen Charakter dieser Erzählungen zu tun. Ihnen sei nicht mit herkömmlichen kritischen Maßstäben beizukommen, wie er in einer Passage ausführt, in der er auch schon andeutet, dass er einen Zusammenhang zwischen der Form und der keineswegs eindeutigen Moral der Erzählungen sieht:

Wären sie [Diderots Erzählungen] bloß witzige Spielwerke, deren uns sonst die französische Litteratur so viele gegeben hat; oder wären sie bloß fade Liebesgeschichten zum Zeitvertreibe fürs Kanapee; oder wäre ihr ganzer Endzweck nur, eine ungezweifelte moralische Wahrheit, die schon neunzigmal eingekleidet worden, zum ein und neunzigsten male wieder umzukleiden: so würde ihre Beurtheilung, wenigstens nach dem gemeinen Schlage, nicht schwer seyn. Man hielte nur das Werk gegen die Regeln, die so manches Lehrbuch für diese Gattung der Dichtkunst festgesetzt, und die Kritik wäre fertig. (E1, 100)

Nun zeichnen sich Diderots Erzählungen aber, wie Engel weiter hellsichtig ausführt, sowohl auf der Ebene der Form wie der Moral durch eine irritierende und faszinierende Offenheit aus, die den Leser gleichsam mit in einen kritischen Prozess involviere:

[...] Diderots Erzehlungen bestehen aus hingeworfenen philosophischen Ideen, die er selbst nur bis zu einem gewissen Punkt entwickelt, über die er nicht ganz deutlich entscheidet, und über die es in der That nicht leicht ist zu entscheiden. Wenn man ihn beurtheilt, so kann man mit Ehren nicht anders, als selbst ein Wort über diese Ideen hinzusetzen, mit ihm gemeinschaftlich an ihrer Entwickelung arbeiten, und zur Entscheidung der vorkommenden Schwierigkeiten, wo nicht den letzten, doch einen nähern Versuch wagen. (E1, 100f.)

Nach dieser allgemeinen Einleitung wendet sich Engel dann zunächst dem Entretien d'un père avec ses enfants zu, der ihm noch gelungener scheint als Les deux amis de Bourbonne - insbesondere wegen seiner »schönern Form« und seines »wichtigern Inhalts« $(\mathrm{E} 1,101)$. Über viele Seiten hinweg zeichnet er dann differenziert nach, wie die zentrale philosophische Frage des Entretien, nämlich, ob man sich in gewissen Fällen über die geltenden Gesetze hinwegsetzen dürfe, von Diderot in einer eigenwilligen und neuen Dialogform entwickelt wird; in einer Form, die den Leser eben zum Mit- und Weiterdenken animiert. Diese Leseraktivierung und Offenheit fasziniert Engel, und er skizziert eine geradezu moderne Diderot-Lektüre - doch dann fällt er unversehens wieder in ältere Kritikmuster zurück, wenn er moniert, es bleibe unbefriedigenderweise am Schluss der Erzählung offen, was der Leser nun wirklich denken soll: 
Was uns [...] an dem Diderotischen Werke mißfällt, ist dieß: es befriediget nicht; es hat Anfang und Mittel, aber kein Ende. Von allen Meynungen nimmt sich keine so durch Billigkeit und Mäßigung heraus, daß wir sie allen übrigen ohne vieles Bedenken vorzögen; keine der Personen ist so ruhig, so von Vorurtheilen uneingenommen, daß wir ein volles Vertrauen zu ihr gewönnen. [...] Wir wissen also nach geendigter Lektüre nicht, woran wir sind; [...]. $(\mathrm{E} 2,287)^{39}$

Hätte Diderot nur für Philosophen geschrieben, wäre diese Offenheit nach Engel noch zu verzeihen, doch da er »für die Welt« $(\mathrm{E} 2,289)$ schreibe, habe er aus zwei Gründen gefehlt. Erstens weil »ohne Vollendung keine Befriedigung « $(\mathrm{E} 2,289)$ erreicht werde und man nicht davon ausgehen könne, dass jeder Leser in der Lage und willens sei, die offenen Fragen selber weiter zu denken. Und zweitens weil die verhandelte Frage - eben ob sich ein Einzelner über das bestehende Gesetz hinwegsetzen dürfe - »so nahe mit Rechtschaffenheit und Tugend verwandt « sei, dass es unverantwortlich wäre, sie für den Leser unbeantwortet zu lassen: »In andern Materien mag es gut seyn, die Menschen zu Skeptikern zu machen, aber schwerlich in dieser« (E2, 289). Damit bricht Engel seine Ausführungen zum Entretien ab - ironischerweise nicht ohne darauf hinzuweisen, dass er nur »einige flüchtige Ideen « habe »hierher werfen wollen, deren weitere Untersuchung und Berichtigung« er »dem Leser überlasse[]« (E2, 289).

Danach wendet er sich Les deux amis de Bourbonne zu, die er in einem ersten Zugriff der Kirchenkritik Diderots subsumiert, denn er glaubt, die Erzählung sei eigentlich nur geschrieben worden, um den Brief des Pfarrers Papin »bey guter Gelegenheit an den Mann zu bringen« und so die Geistlichkeit lächerlich zu machen (E2, 289f.). Dann wendet sich Engel der Form der Erzählung zu, wobei er allerdings alles, was er einleitend zur besonderen Qualität der Offenheit und Prozessualität in Diderots Erzählungen bemerkt hat, vergessen zu haben scheint. Jedenfalls vermag er es in dieser Erzählung nicht zu erkennen. Vielmehr gibt er nur seinem Unverständnis über die komplexe Form Ausdruck - und zeigt dabei, dass er diese auch gar nicht richtig verstanden hat. Denn wie aus seinen Ausführungen hervorgeht, glaubt er, die Erzählerstimme des Anfangs sei identisch mit jener des Schlusskommentars:

${ }^{39}$ Hier und im Folgenden wird mit der Sigle E2 und Seitenzahl verwiesen auf den zweiten Teil von Engels Rezension in: Neue Bibliothek der schönen Wissenschaften und der freyen Künste 16/2 (1774), S. 274-302. 
Was uns mißfällt, ist die Einkleidung [von Les deux amis de Bourbonne], die uns in der That bey der ersten Lektüre ein wenig verwirrt hat. Die Erzehlung fängt, wie die gewöhnlichen an, die man geradezu ans Publikum richtet; man hat schon einen ganzen Absatz gelesen, ehe man aus einer hingeworfenen Anrede sieht, daß es ein Brief ist; die Erzehlung geht fort bis auf einen gewissen Punkt, wo sie abbricht, und ein neuer Brief eingelegt wird, in welchem wieder ein dritter liegt, auf welchen ein vierter die Antwort enthält; dann beschließt endlich wieder der Verfasser in seinem ersten Tone, vergißt seinen Bruder, an den er eigentlich schreibt, und redet wieder wie ein ordentlicher Erzehler mit dem Publikum. (E2, 290)

Anders als beim Entretien zeigt Engel hier also keine Sensibilität für die Form und realisiert nicht, dass diese auch bei Les deux amis de Bourbonne bedeutungstragend ist. Oder genauer: Er ahnt durchaus, dass die Form eine Bedeutung hat, doch er sieht sie als misslungen an und erkennt nicht, dass sie zu eben jener Leseraktivierung beiträgt, die er eingangs so lobend hervorgehoben hat: »Diese Nachläßigkeit [bezüglich der Form] soll vermuthlich Natur seyn; aber dann ist es Herrn Diderot, wie manchen andern, ergangen, die weit eher die Natur würden gefunden haben, wenn sie weniger mühsam darnach gesucht hätten« (E2, 291). Engel will dieser Kritik an der Form freilich nicht zu viel Gewicht beimessen, denn wichtiger als jene sei der »gute[] Endzweck des Werks«, nämlich, "uns die Güte des menschlichen Herzens selbst da entdecken« zu lassen, »wo die die äußerlichen Handlungen tadelhaft und strafbar sind « (E2, 291). Damit schwenkt auch er auf jene Hauptlinie der Rezeption ein, in der es vor allem um Sympathie mit - oder eventuell auch Ablehnung von - Olivier und Felix ging, während das auf Reflexion hin angelegte Spiel der Perspektiven keine weitere Beachtung fand. Im Vordergrund stand für die frühe deutsche Rezeption mithin die Begeisterung für die beiden Freunde als treue Draufgänger, die unkorrumpiert von den Konventionen der Gesellschaft eine natürliche Tugendhaftigkeit vorleben. So gelesen wurde Les deux amis de Bourbonne zu einer wichtigen Inspirationsquelle für die überaus populäre Räuber- und Außenseiterliteratur des ausgehenden 18. und frühen 19. Jahrhunderts - oder wie es Goethe, ironisch zurückblickend auf die Begeisterung seiner Generation für die »wackeren Wilddiebe und Schleichhändler« Diderots, formulierte: »[D]ieses Gesindel hat in der Folge auf dem deutschen Parnaß nur allzu sehr gewuchert« (DuW, 532). 
Die Rezeption von Diderots Erzählung, die ganz über die Identifikation mit den Freunden Felix und Olivier verlief, beruhte aber eigentlich, wie gezeigt, auf einem Missverständnis - jedenfalls partiell. Natürlich ist auch bei Diderot die Lust an der Schilderung seiner beiden >Natur-Kerle unverkennbar, und wie zum Beispiel sein später in die Erzählung eingefügter Abschnitt zur Treue unter den sizilianischen Räubern zeigt, partizipierte er auch selbst an einer frühen Räuberromantik - oder inszenierte diese jedenfalls effektvoll. ${ }^{40}$ Ebenso unbestritten ist, dass die literarische Thematisierung der Welt der Unterprivilegierten und Randständigen eine politische Dimension haben konnte; von dieser sprach ja auch schon Goethe in der zitierten Passage aus Dichtung und Wahrheit. Doch das eigentliche und wichtigere politische Moment von Diderots Erzählung liegt in deren Form. Denn während die bloß inhaltliche Bezugnahme auf die Welt der Räuber, Banditen und Schmuggler nur allzu leicht auch in die Niederungen leicht konsumierbarer und insofern kaum noch widerständiger Trivialliteratur führen konnte, ist im komplexen Aufbau von Les deux amis de Bourbonne ein genuin politisch-gesellschaftlicher Impuls zur Reflexion über die Voraussetzungen moralischen Urteilens angelegt. Für diese politische Semantik der Form war die frühe deutsche Rezeption von Diderots Erzählung freilich nicht empfänglich.

${ }^{40}$ Die Passage ist in der Erzählung Teil des ersten Briefs von Frau von ***. Insofern gehört sie zu deren romantisierender Sichtweise und nicht zu der des Erzählers, der am Schluss ins Spiel kommt, oder zu der Diderots. 
